\title{
Observation of Strongly Exchange-Coupled Triplet Pairs in an Organic Semiconductor
}

\author{
Leah R. Weiss, ${ }^{1}$ Sam L. Bayliss, ${ }^{1}$ Felix Kraffert, ${ }^{2}$ Karl J. Thorley ${ }^{3}$ John E. Anthony, ${ }^{3}$ \\ Robert Bittl, ${ }^{2}$ Richard Friend, ${ }^{1}$ Akshay Rao, ${ }^{1}$ Neil C. Greenham*, ${ }^{1}$ and Jan Behrends*2 \\ ${ }^{1}$ Cavendish Laboratory, J.J. Thomson Avenue, University of Cambridge, Cambridge CB3 OHE, UK \\ ${ }^{2}$ Berlin Joint EPR Lab, Fachbereich Physik, Freie Universität Berlin, D-14195 Berlin, Germany \\ ${ }^{3}$ Department of Chemistry, University of Kentucky, Lexington, KY 40506-0055, USA
}

From biological complexes to devices based on organic semiconductors, spin interactions play a key role in the function of molecular systems. For instance, triplet-pair reactions impact operation of organic light-emitting diodes as well as photovoltaic devices. Conventional models for triplet pairs assume they interact only weakly. Here, using electron spin resonance, we observe long-lived, strongly-interacting triplet pairs in an organic semiconductor, generated via singlet fission. Using coherent spin-manipulation of these two-triplet states, we identify exchange-coupled (spin-2) quintet complexes co-existing with weakly coupled (spin-1) triplets. We measure strongly coupled pairs with a lifetime approaching $3 \mu$ s and a spin coherence time approaching $1 \mu \mathrm{s}$, at $10 \mathrm{~K}$. Our results pave the way for the utilization of high-spin systems in organic semiconductors.

The dynamics of spin-dependent reactions impact organic systems across scales of complexity. In vivo radicalpair recombination has been implicated in the biological mechanism for avian navigation and in photosynthesis, while in organic semiconducting materials triplet spin-reactions can determine efficiencies in light-emitting diodes and photovoltaics $^{1-5}$. One such process, singlet fission, enables efficient production of two triplet excitons from an initially excited singlet state ${ }^{6-8}$. This carrier multiplication process has enabled photovoltaic devices with over $100 \%$ external quantum efficiencies and holds promise as a means of harnessing the solar spectrum more efficiently ${ }^{9,10}$.

Fission proceeds from a photogenerated singlet exciton to an overall spin-zero triplet-pair state, conserving spin and enabling efficient triplet-pair formation. This initial pure singlet state can further decohere into the triplet-pair eigenstates, which we study here. Understanding how these triplet-pair states interact, annihilate, and move is critical for harnessing them in optoelectronic or spintronic applications. The fate of triplet pairs depends not only on their electronic degrees of freedom, but also on their spin properties, such as the pair spin coherence time. To date, spin dynamics of triplet pairs have predominantly been explored passively via photoluminescence experiments ${ }^{11-13}$, which do not allow for direct triplet-pair manipulation.

Spin resonance techniques allow for active spin control but have previously been limited to continuous-wave (cw) studies of triplet pair-states ${ }^{14,15}$, although transient spin resonance has provided insight into triplet-transfer and triplet-charge interactions ${ }^{16,17}$. Here we focus on the early-time behaviour of the non-equilibrium population of tripletpair states formed following singlet fission and before thermalization. We report the observation of exchange-coupled triplet pairs forming pure spin-quintet (total spin $S=2$ ) states. Quintet states have been observed previously, for example in synthetic compounds that utilize directly bonded radical species ${ }^{18}$ or in materials with degenerate ground state orbitals ${ }^{19}$. Here we observe, in the solid-state, a distinct category of quintet formation due to exchange coupling 
of excited-state triplet excitons in an organic semiconductor. Using pulsed and transient electron spin resonance (ESR) to achieve direct spin manipulation (Fig. 1a), we find that these pair states have an ensemble coherence time of $\simeq 900 \mathrm{~ns}$ and a transient spin-polarization lifetime of nearly $3 \mu$ s at $10 \mathrm{~K}$ in solution-processed films.

\section{Spin Resonance of Weakly Coupled Triplet Pairs}

TIPS-tetracene is a solution-processible organic semiconductor which has been shown to undergo efficient singlet fission in the solid state ${ }^{20,21}$. Tetracene is a canonical singlet fission system that has been studied extensively for both its spin and electronic properties ${ }^{11,12,22,23}$. The modifications to tetracene not only make the material viable for scalable processing, but also change the crystal packing which in turn impacts electronic overlap in triplet pair geometries $^{24}$. Its characterization via optical spectroscopy provides evidence for a distinct triplet-pair intermediate state $^{25}$. Here we use time-resolved ESR to probe the spin-character of the states formed in TIPS-tetracene from singlet fission.

We first discuss the temperature-dependent transient ESR (trESR) spectra of spin-coated TIPS-tetracene films taken at 300 ns after laser excitation (see Fig. 1c for spectra, Fig. 1b and Methods for experimental scheme). Spin resonance spectral line shapes for organic triplet pairs depend on the relative strength of the Zeeman interaction, the intratriplet electron-hole dipolar coupling, and the intertriplet spin-coupling, as expressed in the following Hamiltonian ${ }^{26-28}$.

$$
\hat{H}=\underbrace{J \hat{\mathbf{S}}_{a} \cdot \hat{\mathbf{S}}_{b}}_{\text {Exchange }}+\sum_{i=a, b} \underbrace{g \mu_{B} \mathbf{B}_{\mathbf{0}} \cdot \hat{\mathbf{S}}_{i}}_{\text {Zeeman }}+\underbrace{D\left(\hat{S}_{i, z}^{2}-\hat{\mathbf{S}}_{i}^{2} / 3\right)}_{\text {Dipolar }}
$$

As we discuss in more detail below, we take the triplet-triplet interaction to be an isotropic exchange interaction with exchange parameter $J, g$ is the $g$-factor, $\mu_{B}$ the Bohr magneton and the last term describes the intratriplet electronhole dipole-dipole interaction for an axially symmetric triplet with $D$ the zero-field splitting (ZFS) parameter.

At room temperature the trESR spectra are well described by a negligible intertriplet coupling, i.e. $J \ll D$ (Fig. 2a), with polarization due to selective population of the $m_{s}=0$ Zeeman state $|0\rangle$. As we describe below, this selective population arises from the spin dynamics of triplet pairs formed via singlet fission and recombining via triplet-triplet annihilation. Due to the zero-field splitting, the $\left|\Delta m_{s}\right|=1$ emissive $T^{-}$and absorptive $T^{+}$transitions from the $|0\rangle$ state to the $| \pm\rangle$ Zeeman states do not coincide, and these transition peaks are separated in magnetic field by $\Delta B=D / g \mu_{B}$ (Fig. 2c), where the $D$ parameter agrees with previous cw studies ${ }^{15,24}$.

Selective population of the $m_{s}=0$ state through fission can be understood as follows. The triplet-pair state formed by singlet fission conserves spin, forming the overall spin-zero state ${ }^{23,29}$

$$
|S\rangle=\frac{1}{\sqrt{3}}(|00\rangle-|+-\rangle-|-+\rangle)
$$

Since this is a pure singlet state, it has no net magnetic moment and so is unobservable in ESR. However, for weakly coupled pairs, $|S\rangle$ is not an energy eigenstate; instead the eigenstates consist of two singlet-quintet mixtures, four pure spin-quintet states and three pure spin-triplet states ${ }^{23}$. The two singlet-containing eigenstates $\left|\psi_{1}\right\rangle$ and $\left|\psi_{2}\right\rangle$ are 
mixtures of the singlet state $|S\rangle$ and the $m_{s}=0$ quintet state $\left|Q_{0}\right\rangle$ :

$$
\begin{gathered}
\left|\psi_{1}\right\rangle=\sqrt{\frac{1}{3}}|S\rangle+\sqrt{\frac{2}{3}}\left|Q_{0}\right\rangle=|00\rangle \\
\left|\psi_{2}\right\rangle=\sqrt{\frac{2}{3}}|S\rangle-\sqrt{\frac{1}{3}}\left|Q_{0}\right\rangle=\frac{1}{\sqrt{2}}(|+-\rangle+|-+\rangle)
\end{gathered}
$$

In the incoherent limit, the rate of fission and annihilation connecting each pair eigenstate $\left|\psi_{i}\right\rangle$ to the emissive singlet exciton is proportional to its singlet content $\left|\left\langle S \mid \psi_{i}\right\rangle\right|^{2}$ and the relative equilibrium populations of the $|0\rangle$ state is greater than that of the $| \pm\rangle$ states as was first observed in cwESR of tetracene ${ }^{14,30}$ (see Methods for further details). It is this selective population of the $|0\rangle$ state by fission which gives rise to the observed trESR polarization pattern at room temperature (Fig. 2a). We note that we obtained similar spectra for both spin-coated and drop-cast films (see Methods), and that that this pattern cannot arise due to intersystem crossing, which is instead selective in the zero-field basis ${ }^{31,32}$. The polarization pattern thereby provides a means of separating triplet formation mechanisms ${ }^{33}$ and allows us to identify that the trESR signal arises from singlet fission.

\section{Spin Resonance of Exchange-Coupled Triplet Pairs}

As the temperature is lowered, a new set of central peaks emerge. These inner peaks, separated in magnetic field by $D / 3 g \mu_{B}$, i.e. $1 / 3$ of the separation of the outer peaks, are consistent with strongly exchange-coupled triplet pairs $(J>D)$ forming pure $\left|Q_{0}\right\rangle$ quintet states with total spin $S=2$. Fig. 2b shows the trESR spectrum at $10 \mathrm{~K}$ along with a simulation for a combined population of quintet (strongly coupled pairs) co-existing with weakly coupled pairs, showing good agreement with the experimental data. Fig. $2 \mathrm{~d}$ shows the level scheme in the strong exchange coupling regime and corresponding orientation-averaged emissive $Q^{-}$and absorptive $Q^{+}$transitions from $\left|Q_{0}\right\rangle$. This shows that for strongly coupled triplet pairs, the transition peaks from the $\left|Q_{0}\right\rangle$ state have a field separation three times smaller than the weakly coupled transition peaks (Fig. 2c), which can be shown from perturbative calculations treating the intratriplet dipolar coupling as a perturbation on the exchange coupling (Supporting Information) or via transformation between coupled and uncoupled basis representations of the Hamiltonian ${ }^{27}$.

The above analysis is consistent with the interaction between triplets being dominated by exchange coupling rather than dipolar coupling, and we now discuss the physical motivations for this. To achieve the strong-coupling regime observed above - specifically the factor of $1 / 3$ field separation between inner (quintet) and outer (triplet) peaks - the intertriplet coupling strength must be greater than the intratriplet zero-field splitting D $(\sim \mu \mathrm{eV})$. Since the intertriplet dipolar interaction between electron spins cannot exceed the intratriplet interaction, our observations mandate a spin-dependent interaction which can exceed a dipolar interaction, i.e. an exchange interaction. In efficient singlet fission materials the exchange energy approaches half the optical bandgap. Since the intertriplet exchange interaction is thereby limited only by the $\sim \mathrm{eV}$ intra-exciton exchange interaction ${ }^{34}$, this can exceed the dipolar coupling which is limited to $D \sim \mu \mathrm{eV}$. Our results are consistent with a triplet-triplet dipolar interaction which is smaller than the line-broadening and the dominant intertriplet interaction as an isotropic exchange interaction. 
To verify the assignment of the inner peaks to quintet pair states we perform Rabi nutation experiments on the triplet and quintet peaks (Fig. 3). Since the Rabi nutation frequency depends on the total spin of a species (Fig. 3a), this provides an unambiguous assignment of the different transitions ${ }^{35}$. For a state with total spin $S$ being pumped between levels with spin-projections $m_{s}$ and $m_{s} \pm 1$ the Rabi oscillation frequency is

$$
\omega_{m_{s}, m_{s} \pm 1}=\omega_{1 / 2} \sqrt{S(S+1)-m_{s}\left(m_{s} \pm 1\right)}
$$

where $\omega_{1 / 2}=g \mu_{B} B_{1} / \hbar$ and $B_{1}$ is the microwave field strength. For transitions from the $m_{s}=0$ state, the ratio of Rabi frequencies for pure quintet and triplet states becomes $\sqrt{3}$. This therefore provides a direct probe of the spin state of the observed transitions. The triplet transition frequency acts as an intrinsic reference, without the need for an external standard.

Fig. 3b-c shows the Rabi oscillations for the two absorptive transition peaks $\left(T^{+}\right.$and $\left.Q^{+}\right)$along with their Fast Fourier Transform (FFT) for both drop-cast thin films and macrocrystalline TIPS-tetracene samples formed from saturated solutions. Within the error we measure a factor of $\sqrt{3}$ between the oscillation frequency of the inner quintet peak and the outer triplet peak (for fixed $B_{1}$ ) for both samples, confirming that the inner peaks arise from strongly exchange-coupled triplet pairs forming pure quintet states. To our knowledge this measurement provides the first observation of high-spin state formation $(S=2)$ from coupled triplet excitons in an organic semiconductor.

These observations are consistent with time-resolved optical measurements on TIPS-Tetracene films, which show that singlet fission is approximately temperature-independent due to the formation of electronically coupled triplet pair states. ${ }^{20,25}$. These results are also consistent with the detection of ODMR signals due to weakly coupled triplet pairs formed from singlet fission ${ }^{15}$ : since ODMR is sensitive to changes in net singlet content it should preferentially probe weakly coupled (mixed singlet-quintet) states.

\section{Coherence and Lifetime of Exchange-Coupled Triplet Pairs}

The clean separation of the spectral features of quintet states from those of free or weakly coupled triplets gives a selective experimental handle for studying triplet pairs; $S=2$ states can only form from two interacting triplets. We can therefore directly study the properties of these triplet pair states. We first measure the transient spin lifetime - the time over which spin polarization is maintained in trESR. As shown in Fig. 4a, at $10 \mathrm{~K}$ the exchange-coupled pair spin lifetime in films is $2.85 \pm 0.01 \mu \mathrm{s}$, while for weakly interacting triplets this lifetime is $3.88 \pm 0.01 \mu \mathrm{s}$. We note that these ESR transients are a lower bound for the intrinsic spin lifetime, and include effects of relaxation in addition to population dynamics. Note that the rise of both the triplet and quintet signals are within the $\sim 300 \mathrm{~ns}$ instrument response of the transient spectrometer, and the difference in time of the peak signal intensities are due to the difference in decay rates for the two species (see SI).

We next probe the spin coherence of these exchange-coupled triplet pairs. To measure a bound on the intrinsic transverse coherence time $T_{2}$ of both weakly and strongly coupled triplets we use a Hahn echo pulse sequence (Fig 4c) which refocuses any of the reversible contributions to the ensemble dephasing. This sequence uses a $\pi$ pulse to invert 
the phase of the spin ensemble after a dephasing time $\tau$ and thereby rephases the spins following subsequent coherent evolution, which is then measured as an echo peak in ESR intensity at time $2 \tau$ (Fig. 4c) ${ }^{36}$. As the free evolution time $\tau$ increases, more pair states undergo incoherent processes that cannot be reversed. Measuring the echo intensity decay as a function of $\tau$ therefore allows us to extract the coherence times $T_{2}$. Note that these experiments measure dephasing of states with mixed spin projections (e.g., $Q_{0}$ and $Q_{1}$ ).

For the the strongly coupled (quintet) pair at $10 \mathrm{~K}$ we find $T_{2}$ times of $0.87 \pm 0.04 \mu \mathrm{s}$ and $0.84 \pm 0.02 \mu \mathrm{s}$ for the thin film and macrocrystalline sample respectively measured at the $Q^{+}$transition (bottom graph, Fig. 4 b). For the triplet $T^{+}$transition we find $T_{2}$ times of $0.69 \pm 0.04 \mu \mathrm{s}$ and $0.58 \pm 0.01 \mu \mathrm{s}$ for the film and macrocrystalline samples (top graph, Fig. 4 b). The microsecond timescales of spin polarization and coherence indicate that triplets can remain in close proximity and coherent over timescales well beyond those measured in typical optical spectroscopy experiments, which usually focus on fs-ns ranges ${ }^{8}$. Sustained spin coherence over microsecond timescales suggests coherence may be maintained through diffusion, transfer, and dissociation events in devices and could play a role in harnessing triplet pairs formed by singlet fission. The coherence time of quintet-coupled pair states here surpasses that of polaron pairs measured at $10 \mathrm{~K}$ in organic semiconducting films ${ }^{37}$. Indeed, $T_{2}$ approaches the $\sim 1 \mu$ s coherence times measured in diamond nitrogen-vacancy centers at room temperature for high-pressure, high-temperature synthetic diamond ${ }^{38}$, positioning organic triplet spin pairs as a viable source of coherent pair states for applications in quantum information and spintronics.

Engineering longer coherence times requires an understanding of relevant decoherence mechanisms. Determining the dominant mechanisms of decoherence in organic semiconductors presents a current experimental and theoretical challenge. In this system a possible source of decoherence is geometric relaxation or hopping between sites, varying the exchange coupling ${ }^{39}$. Such variation in coupling through time would produce rapid variation in the energetic separation of singlet/quintet pairs, leading to irreversible coherence decay depending on the timescale of these fluctuations. Decoherence may also arise due time-dependent variation in fine structure tensors due to hopping between sites or molecular nutation ${ }^{37}$, or due to hyperfine coupling, although the latter may be suppressed by motional averaging ${ }^{14,40,41}$. In the both the triplet and quintet states, the lack of significant dependence on sample type suggests that decoherence of the pairs has minimal dependence on morphology. Future experiments and theoretical modelling of the temperature-dependence of the competing decoherence mechanisms is a promising avenue for elucidating the physical mechanism of spin decoherence in singlet fission systems.

\section{Quintet Formation and Magnetic Signatures From Singlet Fission in the Strong Coupling Regime}

In the strong-coupling regime we might expect fission to proceed into the ESR-silent pure singlet eigenstate $|S\rangle$, where the pair would remain since $|S\rangle$ is an energy eigenstate for strong exchange coupling. How then do the quintet coupled pair states form? To explain this, we posit a mechanism for quintet formation through two successive decoherence steps mediated by the exchange interaction (Fig. 5). In this model, triplet pairs undergo a variation in exchange coupling through time, enabling a rapid transition from weak to strong coupling regimes as mentioned previously (this may be due to polaronic distortion, molecular relaxation, or hopping to sites with smaller interpair 
separation, for example). Weakly coupled eigenstates are singlet-quintet mixtures (Equation (1a,b)). A rapid increase in $J$ for these states would therefore induce a collapse into the pure spin states $|S\rangle$ and $\left|Q_{0}\right\rangle$ since singlet and quintet states become separated energy eigenstates, thus explaining why the $\left|Q_{0}\right\rangle$ state is selectively populated relative to the other quintet states, as observed experimentally.

As an estimate of the exchange parameter $J$, we fit the temperature dependence of the ratio of triplet to quintet formation with a temperature activation of $4.2 \mathrm{meV}$ (SI Fig. 1). This activation energy acts as an upper bound on $J$, as it includes both the thermal energy required to escape the strongly coupled quintet manifold $\left(k_{B} T>J\right)$ and any polaronic or morphology-dependent activation energy, for example due to hopping out of triplet pair trap sites. We note that while our experiments do not measure $J$ directly, this can be determined using high magnetic field level-anticrossings between the singlet and quintet pair states, which will cause changes in the photoluminescence at the field positions determined by $J^{42,43}$.

The observation of strongly coupled triplet pairs from singlet fission has important consequences for how singlet fission is identified. In weakly coupled pairs, the transition between the zero-field dipolar Hamiltonian and high-field Hamiltonian results in magnetic modulation of photoluminescence (at field strengths $B \sim D / g \mu_{B}$ ) and this is often used as a signature of singlet fission and triplet-triplet annihilation ${ }^{29,44}$. However, for exchange-dominated triplet pairs no such low-magnetic-field effect will be observed. For systems in which exchange coupling is significant, low-field effects alone cannot therefore be used to identify singlet fission, but rather should be combined with spin resonance techniques and high field studies to allow sensitivity to a range of coupling strengths ${ }^{42}$.

\section{Conclusions and Outlook}

Coherent control of high-spin, triplet-pair states, as demonstrated here, opens new avenues for applications of triplet pairs in quantum information and spintronics. Coherence and spin-spin interactions in intermediate pair states also critically impact the rate and outcome of triplet-triplet annihilation and other spin-dependent reactions. By tuning intermediate-pair couplings and coherence properties it may therefore be possible to control the outcomes of such interaction events. The formation of pure singlet and quintet eigenstates separated in energy may enable spin protection either to enhance emission from pure singlet pair states or to protect against radiative decay in pure quintet states, which are typically too low in energy to undergo annihilation into the high-energy single-molecule quintet states ${ }^{45}$. Tuning the sign of $J$ would allow ordering of the spin manifolds and enable tuning between emissive singlet and dark quintet pair states. This may be especially useful for upconversion systems or organic light-emitting diodes relying on efficient triplet-triplet annihilation ${ }^{46,47}$. By contrast, triplet-triplet annihilation can also present a loss pathway in organic photovoltaics and phosphorescent light-emitting diodes and spin-protected quintet states would therefore be beneficial there ${ }^{48,49}$. Beyond the application of exchange for spin-protection, these high-spin coherent states may also prove useful in hybrid organic-inorganic systems. Triplet transfer into inorganic quantum dot systems has been demonstrated to be fast and efficient ${ }^{10,50}$, and the long spin-lifetime and coherence in triplet pair states suggests that coherence may be maintained through processes of diffusion and transfer across interfaces in devices. Combining the intrinsically polarized, coherent triplet-pair states with traditional inorganic systems such 
as GaAs may enable new applications in quantum information and spintronics. Can the quantum phase information generated in an organic material be maintained across interfaces? Understanding the role of coherence and spinspin interactions in organic semiconductors and hybrid materials holds promise for molecular engineering of these properties and opens up new research avenues in optoelectronic, spintronic, and hybrid material systems.

\section{Methods}

\section{Material Preparation}

TIPS-Tetracene thin film samples were either spin-coated or drop cast from $100 \mathrm{mg} / \mathrm{ml}$ chloroform solutions onto quartz substrates in a nitrogen atmosphere $\left(<0.1 \mathrm{ppm}_{2},<0.1 \mathrm{ppm} \mathrm{H}_{2} \mathrm{O}\right)$. Macrocrystalline samples consisting of 1-3 mm-scale domains were crystallized from saturated hexane solution. For all ESR measurements, samples were placed in quartz ESR tubes which were flame-sealed under a helium atmosphere.

\section{Electron Spin Resonance}

Transient ESR was performed using a Bruker MD5 dielectric ring resonator and an X-band ESR spectrometer. Pulsed $532 \mathrm{~nm}$ excitation was provided by a Nd:YAG laser (Atum Laser Titan AC compact $15 \mathrm{MM}$ ) with a 5 ns pulse length. $\mathrm{Cw}$ microwave illumination was applied as the static magnetic field was swept. Pulsed ESR was performed using a Bruker E580 spectrometer configured for X-band and a dielectric resonator (Bruker MD5). Microwave pulses were generated and amplified by a $1 \mathrm{~kW}$ travelling-wave tube amplifier. Pulse sequences were externally triggered by pulsed laser excitation at $532 \mathrm{~nm}$. The Rabi nutation sequence comprised a constant delay after the laser flash $t$ followed by a variable length manipulation pulse, a constant offset, and then echo detection using a $16 \mathrm{~ns} \pi / 2$-pulse followed by a delay (100 ns) then a $32 \mathrm{~ns} \pi$-pulse for the macrocrystalline sample $(t=1.3 \mu \mathrm{s})$ and $16 \mathrm{~ns} \pi$ pulse for the thin film measurements with power adjusted accordingly $(t=1 \mu \mathrm{s})$. The Hahn echo decay experiment sequences used a $\pi / 2$ pulse, variable delay $\tau$ followed by a $\pi$ pulse with measurement of decaying echo intensity as a function of $\tau$. The $\pi / 2$ pulse length was $16 \mathrm{~ns}$ with delay after flash $(t=1.3 \mu \mathrm{s})$ for macrocrystalline measurements, while for thin film measurements the $\pi / 2$ pulse length was $8 \mathrm{~ns}$ with delay after flash $(t=1 \mu \mathrm{s})$.

\section{Spectral Simulation}

Simulations were performed using a stochastic Liouville simulation (similar to Bayliss et al. ${ }^{24}$ ) for coupled triplet pairs. The ESR response as a function of magnetic field is found by solving the following master equation for the first-order ESR susceptibility:

$$
\frac{\partial \rho(t)}{\partial t}=-\frac{i}{\hbar}\left[\hat{H}_{0}+\hat{H}_{1}(t), \hat{\rho}\right]+\hat{G}-\gamma_{d} \hat{\rho}-\frac{1}{2} \gamma_{-}\left\{\hat{P}_{S}, \hat{\rho}\right\}
$$


Here $\hat{\rho}$ is the triplet-pair density matrix, [] the commutator, $\hat{H}_{0}$ is the static Hamiltonian, as defined in Equation (1) of the main text, $\hat{H}_{1}(t)$ is the perpendicular ac microwave Hamiltonian, $\hat{G}(t)$ describes the generation of triplet-pair states via singlet fission according to the singlet projector $\hat{P}_{s}=|S\rangle\langle S|, \gamma_{d}$ is the spin independent decay constant, $\gamma_{-}$ the triplet-pair spin-dependent decay constant via the singlet channel, and \{\} the anticommutator. The ESR signal connecting two spin sublevels is proportional to the magnetic dipole transitions induced by the ac-field and to the population differences between these sublevels. We define the populations used in the spectral simulations below.

\section{Kinetic Population}

At room temperature we find the exchange term in $\hat{H}_{0}$ to be negligible. In this regime, the spin sublevel populations used to fit the room-temperature spectrum were determined from the dynamics of fission, annihlation and spin-independent decay which lead to a build-up of population in the $|0\rangle$ state. We refer to this selective population of the $|0\rangle$ state as kinetic populations. This kinetic population is explained as follows (see also Swenberg et al. ${ }^{30}$ ). The populations, disregarding any off diagonal terms in the incoherent kinetic limit, were calculated according to the following kinetic scheme.

$$
\hat{P}_{s} \underset{\alpha_{i} \gamma_{-}}{\stackrel{\alpha_{i} \gamma_{+}}{\rightleftharpoons}}\left\{\hat{\rho}_{i i}\right\} \stackrel{\gamma_{d}}{\longrightarrow}
$$

where $\alpha_{i}=\left|\left\langle S \mid \psi_{i}\right\rangle\right|^{2}$ as defined in the main text, $\alpha_{i} \gamma_{+}$is the rate of fission to form pair eigenstate $\hat{\rho}_{i i}$, $\alpha_{i} \gamma_{-}$is the rate of pair annihilation to reform the singlet, and $\gamma_{d}$ is the rate of spin-independent decay of triplets. In equilibrium this results in subpopulations given by the following.

$$
\hat{\rho}_{i i}=\frac{\gamma_{+} \alpha_{i} \hat{P}_{s}}{\gamma_{-} \alpha_{i}+\gamma_{d}}
$$

Projecting onto the independent triplet basis states gives a relative population of the $|0\rangle$ and $|+\rangle$ or $|-\rangle$ states of $n_{0} / n_{ \pm}=\frac{\epsilon+2 / 3}{\epsilon+1 / 3}>0$ where $\epsilon=\gamma_{d} / \gamma_{-}$and $n_{i}$ is the population of the single-triplet Zeeman state $|i\rangle$. This explains the selective population of the $|0\rangle$ level by singlet fission and is included in the simulations by setting the $|0\rangle$ level population greater than that of the $| \pm\rangle$ levels. Note that the since the ESR intensities are not quantitative, the precise ratio of $n_{0} / n_{ \pm}$is not important since this just acts to scale the spectrum and does not influence the lineshape.

\section{Quintet Population}

The $10 \mathrm{~K}$ simulations include quintet $\left|Q_{0}\right\rangle$ population with $J \gg D$ and population constrained to the $\left|Q_{0}\right\rangle$ quintet sublevel with $m_{s}=0$. 
Thermal Population

A subpopulation of thermally-generated polarization was also included following a Boltzmann distribution with the same Hamiltonian parameters, which accounts for the slight asymmetry in the low field and high field transitions (the kinetic model is symmetric with respect to the absorptive and emissive transitions). In this case the population follows the Boltzmann distribution below where $\omega_{n}=E_{n} / \hbar$ is determined by the energy of the $n$-th pair

$$
\hat{\rho}_{i i}=\frac{\exp \left(-\hbar \omega_{n} / k_{B} T\right)}{\sum_{m} \exp \left(-\hbar \omega_{m} / k_{B} T\right)} .
$$

For spectral simulations at $300 \mathrm{~K}$ we use $D / g \mu_{B}=48 \mathrm{mT}$ and $J \ll D$ with a 0.8 weight of kinetic populations and a 0.2 weight of thermal triplet populations to account for the slight asymmetry in low and high field transitions. For simulations for $10 \mathrm{~K}$ spectra, we use $D / g \mu_{B}=50 \mathrm{mT}$ and $J=100 D$ (satisfying $J \gg D$ ) for the quintet $\left|Q_{0}\right\rangle$ component (weight 0.8 ), and $J=10^{-3} D$ for the weak-coupling component (0.6 kinetic and 0.2 thermal population weights).

1 Ritz, T., Thalau, P., Phillips, J. B., Wiltschko, R. \& Wiltschko, W. Resonance effects indicate a radical-pair mechanism for avian magnetic compass. Nature 429, 177-180 (2004).

2 Baker, W., McCamey, D., Van Schooten, K., Lupton, J. M. \& Boehme, C. Differentiation between polaron-pair and tripletexciton polaron spin-dependent mechanisms in organic light-emitting diodes by coherent spin beating. Phys. Rev. B 84, 165205 (2011).

${ }^{3}$ Lubitz, W., Lendzian, F. \& Bittl, R. Radicals, radical pairs and triplet states in photosynthesis. Acc. Chem. Res. 35, 313-320 (2002).

${ }^{4}$ Rao, A. et al. The role of spin in the kinetic control of recombination in organic photovoltaics. Nature 500, 435-439 (2013).

${ }^{5}$ Nguyen, T. D., Ehrenfreund, E. \& Vardeny, Z. V. Spin-polarized light-emitting diode based on an organic bipolar spin valve. Science 337, 204-209 (2012).

${ }^{6}$ Swenberg, C. E., Geacintov, N. \& Birks, J. Excitonic interactions in organic solids (1973).

7 Smith, M. B. \& Michl, J. Singlet fission. Chem. Rev. 110, 6891-6936 (2010).

8 Smith, M. B. \& Michl, J. Recent advances in singlet fission. Annu. Rev. Phys. Chem. 64, 361-386 (2013).

${ }^{9}$ Congreve, D. N. et al. External quantum efficiency above $100 \%$ in a singlet-exciton-fission-based organic photovoltaic cell. Science 340, 334-337 (2013).

10 Tabachnyk, M., Ehrler, B., Bayliss, S., Friend, R. H. \& Greenham, N. C. Triplet diffusion in singlet exciton fission sensitized pentacene solar cells. Appl. Phys. Lett. 103, 153302 (2013).

11 Burdett, J. J. \& Bardeen, C. J. Quantum beats in crystalline tetracene delayed fluorescence due to triplet pair coherences produced by direct singlet fission. J. Am. Chem. Soc. 134, 8597-8607 (2012).

12 Burdett, J. J., Piland, G. B. \& Bardeen, C. J. Magnetic field effects and the role of spin states in singlet fission. Chem. Phys. Lett. 585, 1-10 (2013).

13 Wang, R. et al. Magnetic dipolar interaction between correlated triplets created by singlet fission in tetracene crystals. Nat. commun. 6 (2015). 
14 Yarmus, L., Rosenthal, J. \& Chopp, M. Epr of triplet excitions in tetracene crystals: spin polarization and the role of singlet exciton fission. Chem. Phys. Lett. 16, 477-481 (1972).

15 Bayliss, S. L. et al. Geminate and nongeminate recombination of triplet excitons formed by singlet fission. Phys. rev. lett. 112, 238701 (2014).

16 Chernick, E. T. et al. Pentacene appended to a tempo stable free radical: The effect of magnetic exchange coupling on photoexcited pentacene. J. Am. Chem. Soc. 137, 857-863 (2015).

17 Mauck, C. M., Brown, K. E., Horwitz, N. E. \& Wasielewski, M. R. Fast triplet formation via singlet exciton fission in a covalent perylenediimide- $\beta$-apocarotene dyad aggregate. J. Phys. Chem. A 119, 5587-5596 (2015).

18 Teki, Y., Miyamoto, S., Iimura, K., Nakatsuji, M. \& Miura, Y. Intramolecular spin alignment utilizing the excited molecular field between the triplet $(s=1)$ excited state and the dangling stable radicals $(s=1 / 2)$ as studied by time-resolved electron spin resonance: Observation of the excited quartet $(\mathrm{s}=3 / 2)$ and quintet $(\mathrm{s}=2)$ states on the purely organic $\pi$-conjugated spin systems. Journal of the American Chemical Society 122, 984-985 (2000).

19 Murai, H., Safarik, I., Torres, M. \& Strausz, O. P. Triplet ground-state benzoylphenylmethylene and its quintet ground-state triplet-triplet radical pair. Journal of the American Chemical Society 110, 1025-1032 (1988).

20 Stern, H. Private correspondence .

${ }^{21}$ Odom, S. A., Parkin, S. R. \& Anthony, J. E. Tetracene derivatives as potential red emitters for organic leds. Org. lett. 5, 4245-4248 (2003).

22 Burdett, J. J., Gosztola, D. \& Bardeen, C. J. The dependence of singlet exciton relaxation on excitation density and temperature in polycrystalline tetracene thin films: Kinetic evidence for a dark intermediate state and implications for singlet fission. J. Chem. Phys. 135, 214508 (2011).

${ }^{23}$ Merrifield, R., Avakian, P. \& Groff, R. Fission of singlet excitons into pairs of triplet excitons in tetracene crystals. Chem. Phys. Lett. 3, 386-388 (1969).

${ }^{24}$ Bayliss, S. L. et al. Localization length scales of triplet excitons in singlet fission materials. Phys. Rev. B 92, 115432 (2015).

${ }^{25}$ Stern, H. L. et al. Identification of a triplet pair intermediate in singlet exciton fission in solution. Proc. Natl. Acad. Sci. 112, 7656-7661 (2015).

${ }^{26}$ Schweiger, A. \& Jeschke, G. Principles of pulse electron paramagnetic resonance (Oxford University Press on Demand, 2001).

27 Bencini, A. \& Gatteschi, D. Electron paramagnetic resonance of exchange coupled systems (Springer Science \& Business Media, 2012).

${ }^{28}$ Keevers, T. \& McCamey, D. Theory of triplet-triplet annihilation in optically detected magnetic resonance. Phys. Rev. B 93, 045210 (2016).

29 Johnson, R. \& Merrifield, R. Effects of magnetic fields on the mutual annihilation of triplet excitons in anthracene crystals. Phys. Rev. B 1, 896 (1970).

30 Swenberg, C., Van Metter, R. \& Ratner, M. Comments on exciton fission and electron spin resonance in tetracene single crystals. Chem. Phys. Lett. 16, 482-485 (1972).

31 Budil, D. E. \& Thurnauer, M. C. The chlorophyll triplet state as a probe of structure and function in photosynthesis. BBA-BIOENERGETICS 1057, 1-41 (1991).

32 El-Sayed, M., Leung, M. \& Lin, C. Pmdr spectroscopy and the geometry of the triplet state. Chem. Phys. Lett. 14, 329-334 (1972).

${ }^{33}$ Kraffert, F. et al. Charge separation in pcpdtbt: Pcbm blends from an epr perspective. J. Phys. Chem. C 118, 28482-28493 
(2014).

${ }^{34}$ Wilson, J. S. et al. The energy gap law for triplet states in pt-containing conjugated polymers and monomers. Journal of the American Chemical Society 123, 9412-9417 (2001).

35 Astashkin, A. \& Schweiger, A. Electron-spin transient nutation: a new approach to simplify the interpretation of esr spectra. Chem. phys. letters 174, 595-602 (1990).

36 Hahn, E. L. Spin echoes. Phys. rev. 80, 580 (1950).

37 Baker, W., Keevers, T., Lupton, J. M., McCamey, D. \& Boehme, C. Slow hopping and spin dephasing of coulombically bound polaron pairs in an organic semiconductor at room temperature. Phys. rev. lett. 108, 267601 (2012).

38 Rondin, L. et al. Magnetometry with nitrogen-vacancy defects in diamond. Rep. Prog. Phys. 77, 056503 (2014).

39 McLauchlan, K. A. \& Steiner, U. Invited article: The spin-correlated radical pair as a reaction intermediate. Mol. Phys. 73, 241-263 (1991).

40 Deigen, M. \& Pekar, S. Hyperfine interactions and spin-electron resonance in polarons and excitons. Soviet Physics Jetp 34 (1958).

41 Sternlicht, H. \& McConnell, H. M. Paramagnetic excitons in molecular crystals. The Journal of Chemical Physics 35, 1793-1800 (1961).

42 Bayliss, S. L. et al. Spin signatures of exchange-coupled triplet pairs formed by singlet fission. Physical Review B 94, 045204 (2016).

43 Wakasa, M. et al. What can be learned from magnetic field effects on singlet fission: Role of exchange interaction in excited triplet pairs. J. Phys. Chem. C 119, 25840-25844 (2015).

44 Steiner, U. E. \& Ulrich, T. Magnetic field effects in chemical kinetics and related phenomena. Chem. Rev. 89, 51-147 (1989).

45 Dick, B. \& Nickel, B. Accessibility of the lowest quintet state of organic molecules through triplet-triplet annihilation; an indo ci study. Chem. Phys. 78, 1-16 (1983).

46 Singh-Rachford, T. N. \& Castellano, F. N. Photon upconversion based on sensitized triplet-triplet annihilation. Coord. Chem. Rev. 254, 2560-2573 (2010).

47 Kondakov, D., Pawlik, T., Hatwar, T. \& Spindler, J. Triplet annihilation exceeding spin statistical limit in highly efficient fluorescent organic light-emitting diodes. J. Appl. Phys. 106, 124510 (2009).

${ }^{48}$ Baldo, M. A., Adachi, C. \& Forrest, S. R. Transient analysis of organic electrophosphorescence. II. transient analysis of triplet-triplet annihilation. Phys. Rev. B 62, 10967 (2000).

49 Reineke, S., Schwartz, G., Walzer, K. \& Leo, K. Reduced efficiency roll-off in phosphorescent organic light emitting diodes by suppression of triplet-triplet annihilation. Appl. Phys. Lett. 91, 123508 (2007).

50 Thompson, N. J. et al. Energy harvesting of non-emissive triplet excitons in tetracene by emissive pbs nanocrystals. Nature mater. 13, 1039-1043 (2014).

\section{Acknowledgements}

L.R.W. thanks the Gates-Cambridge Trust and Winton Programme for the Physics of Sustainability. This work was supported by the Freie Universität Berlin within the Excellence Initiative of the German Research Foundation. We also acknowledge support from the Engineering and Physical Sciences Research Council [Grants No. EP/G060738/1]. We thank A. D. Chepelianskii for helpful input. The data underlying this paper are available at 
http://dx.doi.org/10.17863/CAM.1634.

\section{Author Contributions}

L.R.W. and S.L.B. analysed the data. F.K., J.B., L.R.W., and S.L.B. carried out the experiments. K.J.T. and J.E.A. provided the materials. All authors discussed the results. L.R.W. and S.L.B. wrote the manuscript with input from all authors.

\section{Competing Financial Interests}

The authors declare no competing financial interests.

\section{Correspondence}

Correspondence regarding material requests should be directed to J.E.A. and all other correspondence regarding the experiments should be sent to J.B. or N.C.G.

\section{Figure Captions}
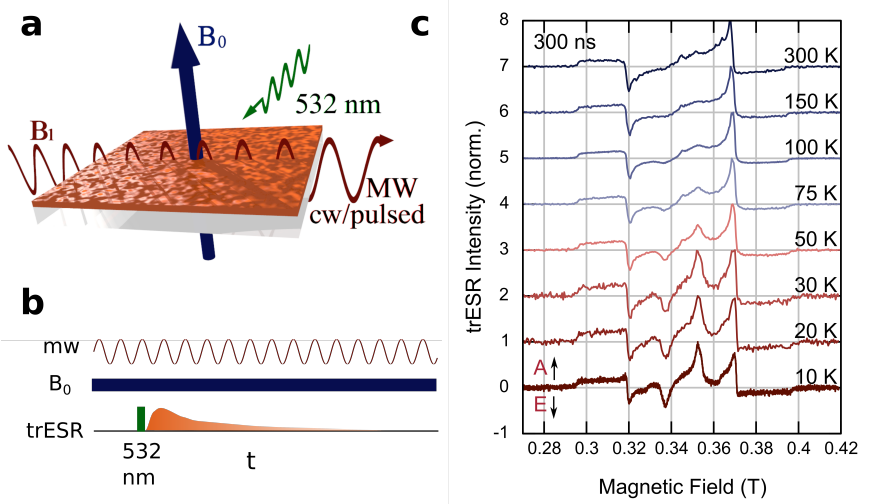

Figure 1: Temperature-dependent formation of exchange-coupled triplet pairs. (a) Simplified schematic of the electron spin resonance (ESR) experiments: TIPS-tetracene thin-film samples are placed in a $9.7 \mathrm{GHz}$ microwave resonator and excited by a pulsed $532 \mathrm{~nm}$ laser in an external field $B_{0}$ perpendicular to the microwave field $B_{1}$. (b) Schematic of transient ESR (trESR) experiments. For each value of the external field, the ESR intensity is recorded as a function of time $t$ following pulsed laser excitation, with constant applied microwave illumination. (c) Formation of interacting triplet pairs. trESR spectral slices as a function of temperature exhibiting a pattern of alternating absorptive (A) and emissive (E) polarization (unless otherwise mentioned spectral slices are taken at $t=300 \mathrm{~ns}$ ). At high temperatures the spectra result from weakly-interacting triplet pairs with polarization in the $m_{s}=0$ state due to singlet fission and triplet-pair annihilation. At low temperatures, the additional inner emissive-absorptive features arise from exchange-coupled triplet pairs $(S=2)$ polarized in the $m_{s}=0$ state. 

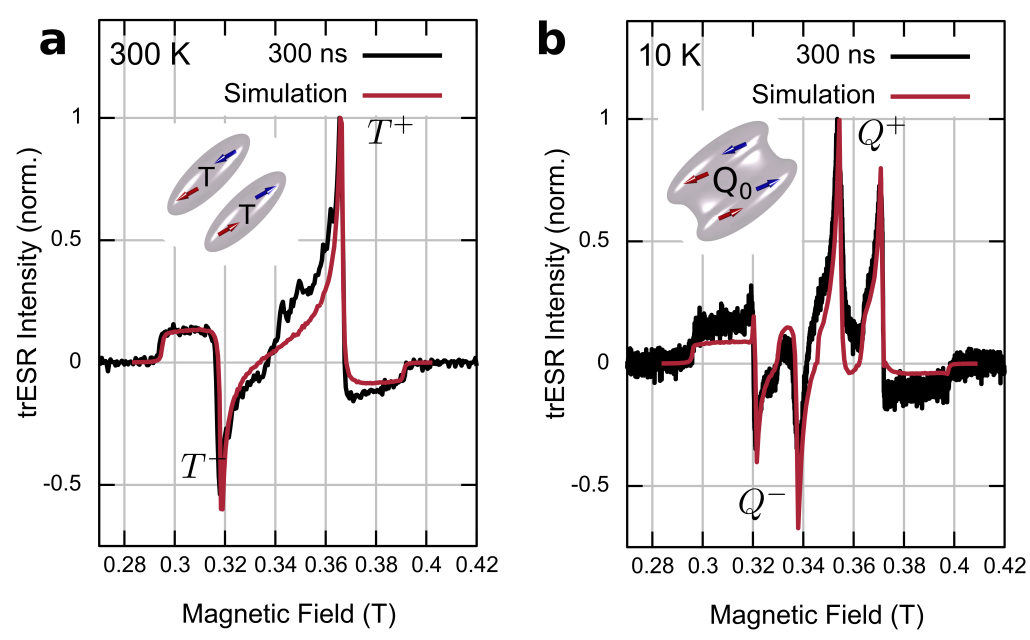

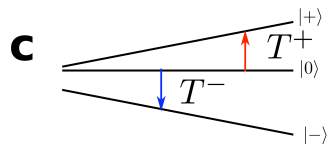

Weak Exchange
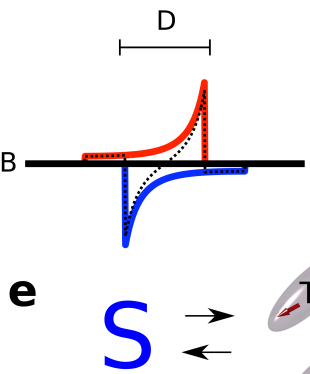

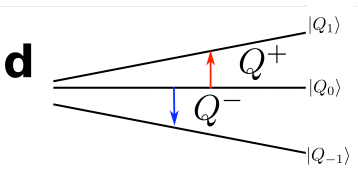

Strong Exchange
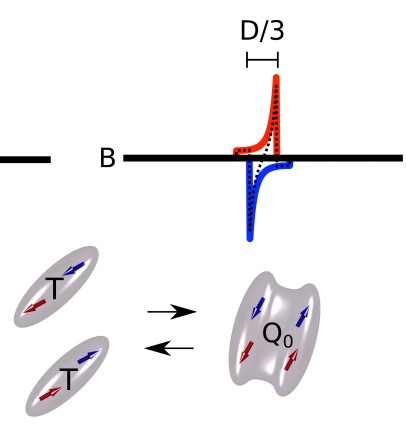

Figure 2: Strongly and weakly coupled pairs formed by singlet fission in TIPS-Tetracene thin-film samples. (a) Room temperature ESR spectrum at $300 \mathrm{~ns}$ along with a fit to a non-interacting triplet $(J=0)$ spectrum, with $D / g \mu_{B}=48 \mathrm{mT}$. $(\mathrm{b})$ trESR spectrum at $10 \mathrm{~K}$. The simulation is for a superposition of strongly-interacting triplet pairs with intertriplet exchange coupling $J \gg D$ and weakly interacting pairs $(J \ll D)$ where $D / g \mu_{B}=50 \mathrm{mT}$. (c) ESR transitions of weakly coupled pairs. Top: Triplet sublevel $(|+\rangle,|0\rangle,|-\rangle)$ energies as a function of field. Arrows denote the absorptive $T^{+}$and emissive $T^{-}$transitions at a constant microwave frequency for molecular fine structure z-axis orientations perpendicular to the field $\left(\mathbf{Z} \perp \mathbf{B}_{\mathbf{0}}\right)$. Bottom: Powder pattern (average over all orientations) for the two transitions. Peaks correspond to the $T^{ \pm}$transitions where $\mathbf{Z} \perp \mathbf{B}_{\mathbf{0}}$. (d) ESR transitions for strongly coupled pairs. Top: Quintet pair-state sublevels $\left|Q_{0}\right\rangle,\left|Q_{+1}\right\rangle$, and $\left|Q_{-1}\right\rangle$ as a function of magnetic field for $\mathbf{Z} \perp \mathbf{B}_{\mathbf{0}}$. Arrows correspond to the $Q^{ \pm}$transitions $\left|Q_{0}\right\rangle \rightarrow\left|Q_{ \pm 1}\right\rangle$. Bottom: Powder average of transitions from the $\left|Q_{0}\right\rangle$ level. Peaks correspond to the $Q^{ \pm}$transitions where $\mathbf{Z} \perp \mathbf{B}_{\mathbf{0}}$. (e) Kinetic model of spin polarization. Transitions between singlet, triplet-pair, and $m_{s}=0$ quintet states. 

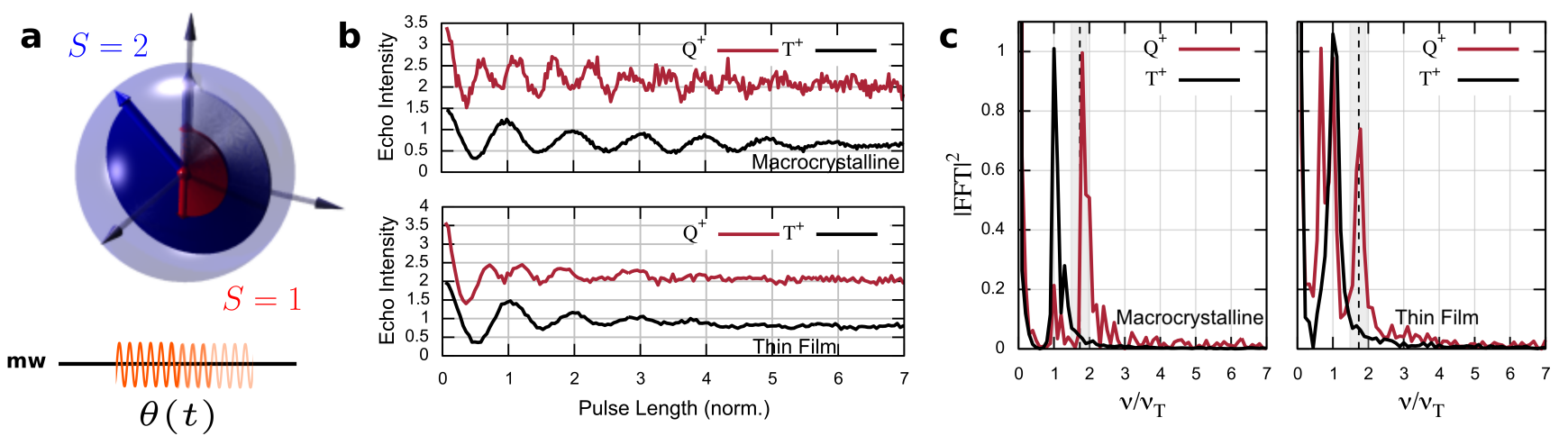

Figure 3: Rabi oscillations of weakly and strongly interacting triplets. (a) Vector model representation of the dependence of the Rabi nutation on total spin in which the nutation frequency increases with $S$. (b) Echo intensity measured as a function of initial pulse length (Rabi oscillations) resulting in distinct oscillation frequencies at the peak triplet $\left(T^{+}\right)$and quintet $\left(Q^{+}\right)$ transitions in both the thin film (bottom) and macrocrystalline samples (top). (c) Fast Fourier Transform (FFT) of Rabi oscillations in (b) revealing a frequency component at the the quintet peak corresponding to a factor of $\sqrt{3}$ higher frequency than the triplet transition, within the error shown in grey. This frequency corresponds to the $\Delta m_{S}= \pm 1$ transitions of the $\left|Q_{0}\right\rangle$ pair state. Note that peak amplitudes are normalized, and that the $Q^{+}$transition in the disordered film also exhibits a Fourier component corresponding to an $S=1 / 2$ species $\left(\nu / \nu_{T}=1 / \sqrt{2}\right)$ due to the presence of non-light-induced charges as well as an $S=1$ species due to overlapping transitions of weakly-coupled triplet states (see SI for additional spin-1/2 frequency comparison). 


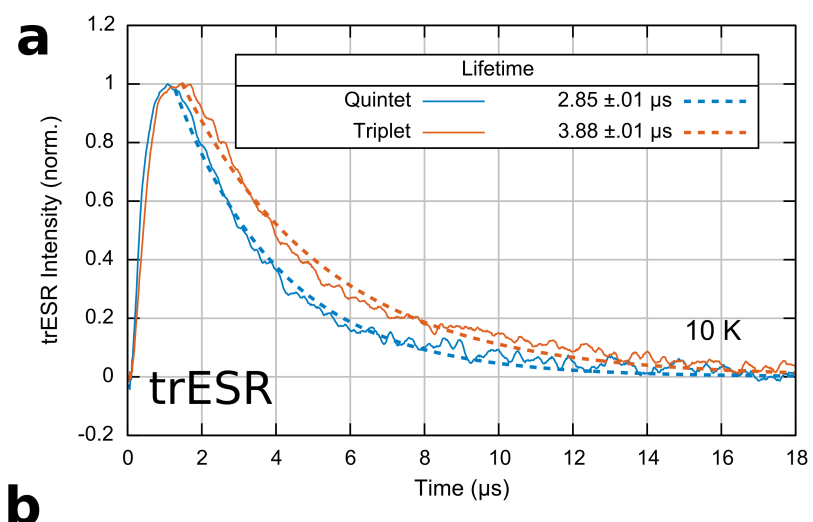

b
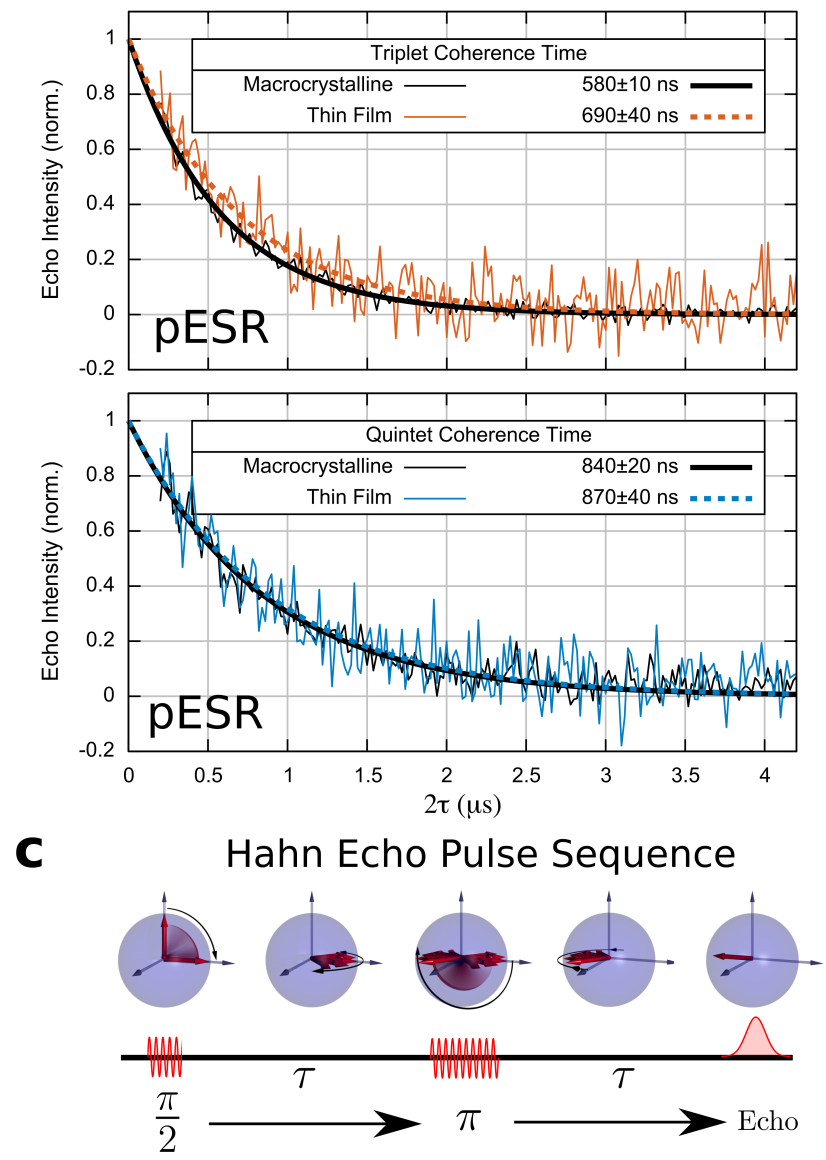

Figure 4: Lifetime and coherence of strongly and weakly coupled triplet pairs. (a) Polarization lifetime of transitions corresponding to quintet and triplet spectral features exhibiting decay with a lifetime of $2.85 \pm .01 \mu$ s and $3.88 \pm .01 \mu$ s respectively. (b) Echo intensity as a function of $2 \tau$ at $10 \mathrm{~K}$. Top: Echo decay measured at the absorptive $T^{+}$triplet peak in a drop-cast thin film $\left(T_{2}=690 \pm 40 \mathrm{~ns}\right.$, orange dashed line) and macrocrystalline sample $\left(T_{2}=580 \pm 10 \mathrm{~ns}\right.$, black solid line $)$. Bottom: Echo decay measured at the absorptive $Q^{+}$quintet peak in both a drop-cast thin film $\left(T_{2}=870 \pm 40 \mathrm{~ns}\right.$, blue dashed line $)$ and macrocrystalline sample $\left(T_{2}=840 \pm 20 \mathrm{~ns}\right.$, black solid line).(c) Vector model representation and pulse schematic of the Hahn echo sequence. The echo intensity is measured as a function of the total free evolution time $2 \tau$. 


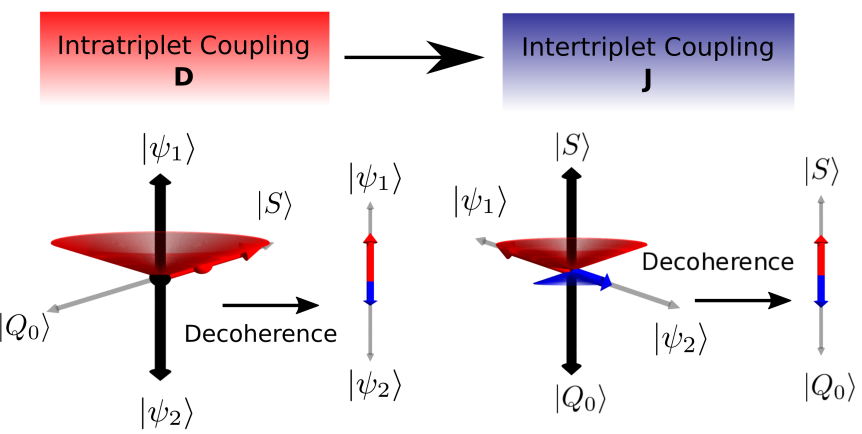

Figure 5: Quintet formation through time-dependent exchange interaction. A time-dependent exchange coupling enables decoherence from the weak-exchange singlet/quintet pair eigenstates $\left|\psi_{1}\right\rangle$ and $\left|\psi_{2}\right\rangle$ into the strong-exchange-coupled quintet $\left|Q_{0}\right\rangle$ eigenstate. The transition from a weak to a strong exchange interaction drives the formation of the pure quintet pair state $\left|Q_{0}\right\rangle$ 


\section{Supporting Information: Observation of Strongly Exchange-Coupled Triplet Pairs in}

an Organic Semiconductor

Leah R. Weiss, ${ }^{1}$ Sam L. Bayliss, ${ }^{1}$ Felix Kraffert, ${ }^{2}$ Karl J. Thorley,${ }^{3}$ John E. Anthony ${ }^{3}$ Robert Bittl, ${ }^{2}$ Richard Friend, ${ }^{1}$ Akshay Rao, ${ }^{1}$ Neil C. Greenham, ${ }^{1}$ and Jan Behrends ${ }^{2}$

${ }^{1}$ Cavendish Laboratory, J.J. Thomson Avenue, University of Cambridge, Cambridge CB3 OHE, UK

${ }^{2}$ Berlin Joint EPR Lab, Fachbereich Physik, Freie Universität Berlin, D-14195 Berlin, Germany

${ }^{3}$ Department of Chemistry, University of Kentucky, Lexington, KY 40506-0055, USA 


\section{THE QUINTET HAMILTONIAN REPRESENTATION AND STRONG EXCHANGE COUPLING}

In the main text, we use the terms strongly coupled triplet pairs and quintet states to describe the interacting triplet pairs. This is because they are two equivalent descriptions of strongly exchange-coupled triplet pairs in the $S=2$ manifold. In the main text and simulations, we use the triplet-pair Hamiltonian $\hat{H}_{0}$, however in the case of strong exchange-coupling the $S=2$ pair states can be equivalently modeled with the following pure quintet Hamiltonian $\hat{H}_{Q}$ where the relation between quintet dipolar hamiltonian and the individual triplet dipolar coupling is given by $D_{Q}=D / 3$ where now $\hat{\mathbf{S}}$ is the quintet spin operator.

$$
\hat{H}_{Q}=\underbrace{g \mu_{B} \mathbf{B}_{\mathbf{0}} \cdot \hat{\mathbf{S}}}_{\text {Zeeman }}+\underbrace{D_{Q}\left(\hat{S}_{Z}^{2}-\hat{\mathbf{S}}^{2} / 3\right)}_{\text {Dipolar }}
$$

The correspondence between strongly coupled triplet pairs each with intratriplet dipolar coupling determined by $D$ and the $S=2$ quintet Hamiltonian with intraquintet dipolar coupling $D_{Q}=D / 3$ can either be taken from full transformation between coupled and uncoupled representations ${ }^{1,2}$ or by a perturbative solution for the effect of intratriplet coupling on the transition energies of strongly exchange-coupled triplet pairs in the quintet eigenstates. Note that we assume collinear dipolar coupling tensors. Taking the dipolar coupling term in $\hat{H}_{0}$ as a perturbation on the intertriplet exchange term, to first order the energy-level differences are given by:

$$
\left|\Delta E_{Q_{0}, Q_{ \pm 1}}\right|=g \mu_{B} B \pm \frac{D}{2}\left(\cos ^{2}(\theta)-\frac{1}{3}\right)
$$

where $\theta$ is the angle of the $\hat{Z}$-axis of the zero-field splitting tensor with the external magnetic field $B_{0}$, while for an individual triplet the energy difference is given by the following

$$
\left|\Delta E_{t_{0}, t_{ \pm 1}}\right|=g \mu_{B} B \pm \frac{3 D}{2}\left(\cos ^{2}(\theta)-\frac{1}{3}\right)
$$

The quintet transitions are therefore equivalent to those of independent triplets but with a factor of 3 reduction in the effective $D$ parameter which gives rise to the factor of three in magnetic field width between the inner and outer peaks observed experimentally. The quintet spectra in our simulations was calculated in the triplet pair basis as described in the Methods with selective population in the $\left|Q_{0}\right\rangle$ sublevel (equivalent to simulations using the open-source software Easyspin ${ }^{3}$ with a quintet $(S=2)$ Hamiltonian and $\left.D_{Q}=D / 3\right)$.

\section{TEMPERATURE ACTIVATED QUINTET DECAY}

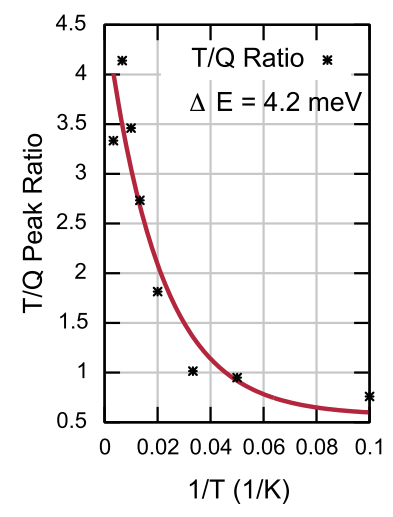

Figure 1. Ratio of absorptive triplet $\mathrm{T}$ and quintet $\mathrm{Q}$ transition intensity as a function of temperature. The extracted activation energy from an Arrhenius equation is $4.2 \mathrm{meV}$.

The ratio of triplet absorptive transition peak height at $T^{+}$to quintet absorption peak height at $Q^{+}($see Figure 2a,b in the main text for spectra and marked field positions) was measured as a function of temperature and fit to an Arrhenius equation to extract the temperature activated quintet decay as shown in Supplementary Figure 1. 


\section{RISE-TIME AND PEAK POSITION OF TRANSIENT ESR KINETICS}

As mentioned in the text, the relative peak positions of the quintet and triplet trESR kinetics arise not due to an intrinsic difference in rise-time, but rather due to instrument limited rise time $(\sim 0.3 \mu \mathrm{s})$ which is the same for both transitions and the faster decay rate of the quintet compared to the triplet transition. The instrument response is determined by the Q-factor of the resonator and the bandwidth of the amplifier. For the critically coupled MD5 resonator the Q-factor is $\gtrsim 5000$ and the amplifier bandwidth is $5 \mathrm{MHz}$. This translates to a $\sim 300 \mathrm{~ns}$ rise time at the X-band $(9.6 \mathrm{GHz})$ frequency used. We show a contour plot and kinetic/spectral traces at early times, which show that as expected from the instrument-response limited rise time, the $300 \mathrm{~ns}$ traces shown in the main text are representative of the earliest time spectra and no spectral shifts are observed prior to 300 ns (SI Figure 2).

\section{a}

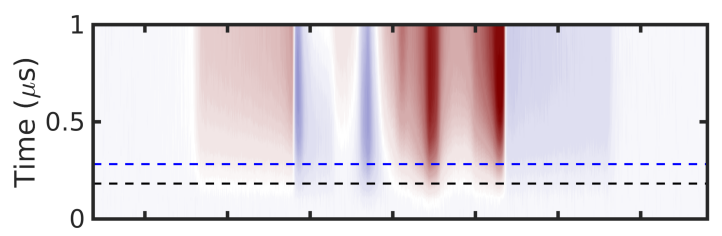

b
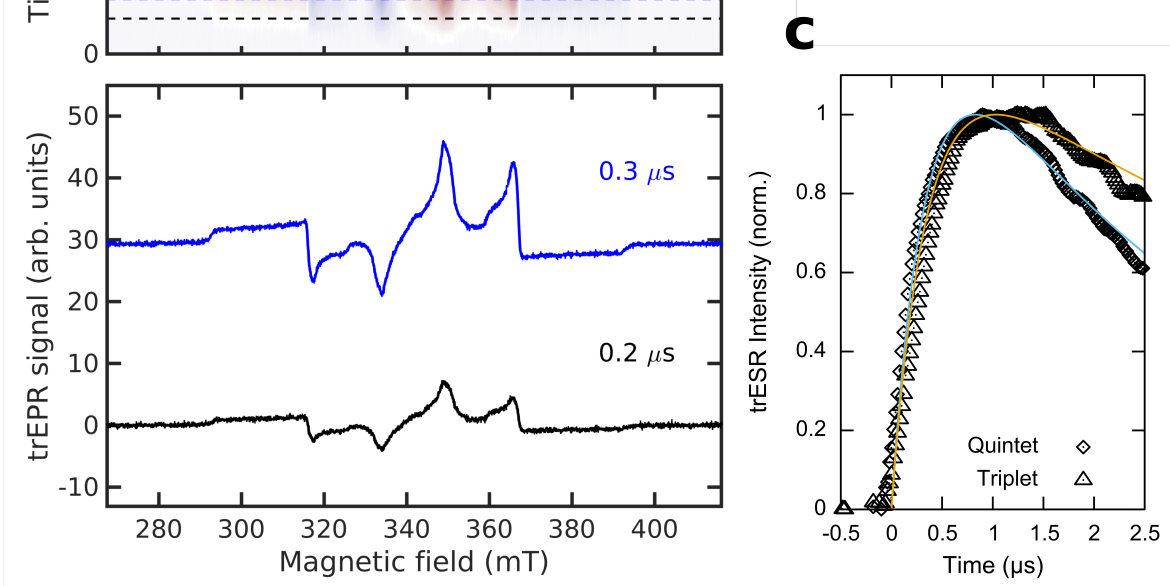

Figure 2. Early time rise of quintet and triplet peaks within the instrument response (300 ns) at $10 \mathrm{~K}$ shown in (a) contour, (b) spectral slices, and (c) kinetic slices with kinetic fit to rise time of $0.3 \mu$ s and different decay rates for the quintet and triplet transitions, as presented in the main text.

\section{SPIN 1/2 REFERENCE NUTATION ANALYSIS}

As described in the main text, the nutation frequency enables spectral assignment of ESR transitions to spin multiplicities as the nutation frequency is related to the $S, m$ transition by the following matrix element.

$$
\omega_{m_{s}, m_{s} \pm 1}=\omega_{1 / 2} \sqrt{S(S+1)-m_{s}\left(m_{s} \pm 1\right)}
$$

In the main text we present confirmation of the $m=0$ quintet character of the additional ESR peaks by comparing the frequency of the triplet transitions with the quintet transitions, which are related by the expected factor of $\nu_{Q_{0}} / \nu_{T}=\sqrt{3}$. As an extra confirmation we can further compare the triplet and quintet transition frequencies to that of a non-light induced charge dopant found in TIPS-Tetracene films, which confirms that $\nu_{1 / 2} / \nu_{T}=1 / \sqrt{2} \sim .7$. See Figure 3 for the nutation traces in time and the corresponding FFT, which indeed reveals the expected frequency ratios. 


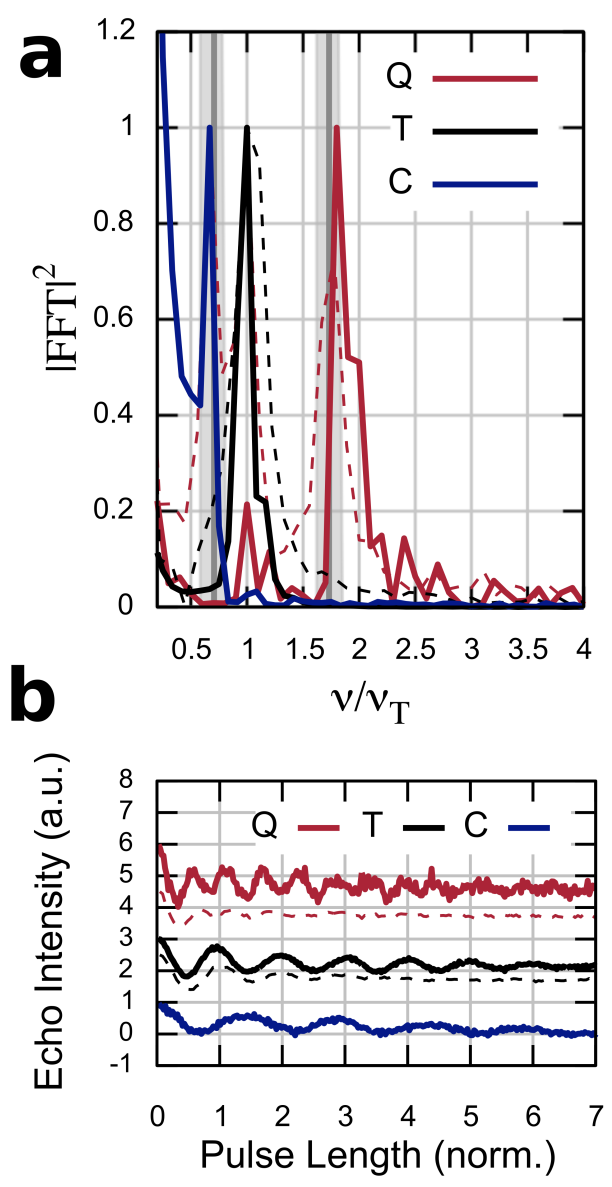

Figure 3. (a)Normalized Fast Fourier Transform, $|\mathrm{FFT}|^{2}$ of time traces displayed in (b) with reference lines corresponding to $1 / \sqrt{2}$ and $\sqrt{3}$, the expected relative transition frequencies of the charge (C) and quintet (Q) transitions relative to the triplet (T) transitions. The triplet and quintet FFT are displayed for the film (dashed) and macrocrystalline sample (solid) and the charge transition is displayed in blue. (b) Raw time traces corresponding to the FFT displayed in (a).

\section{TRANSIENT ESR FLUENCE DEPENDENCE}

The trESR kinetics are displayed as a function of laser intensity for the quintet $\left(Q^{+}\right)$and triplet $\left(T^{+}\right)$ transitions in Figure 4. While there is a faster decay in both cases at higher fluence, no effect is observed on the signal at early times within the noise. Further, the peak trESR signal displays a sub-linear intensity dependence, in keeping with a geminate formation mechanism. The sub-linear behavior of the peak signal is consistent with geminate formation of triplet pairs with contributions of non-geminate annihilation in the decay kinetic, as observed in the increase in trESR decay with power. These results agree with with previous measurements on this material ${ }^{4}$. The sublinear fluence dependence was observed in both macrocrystalline and thin-film samples. 

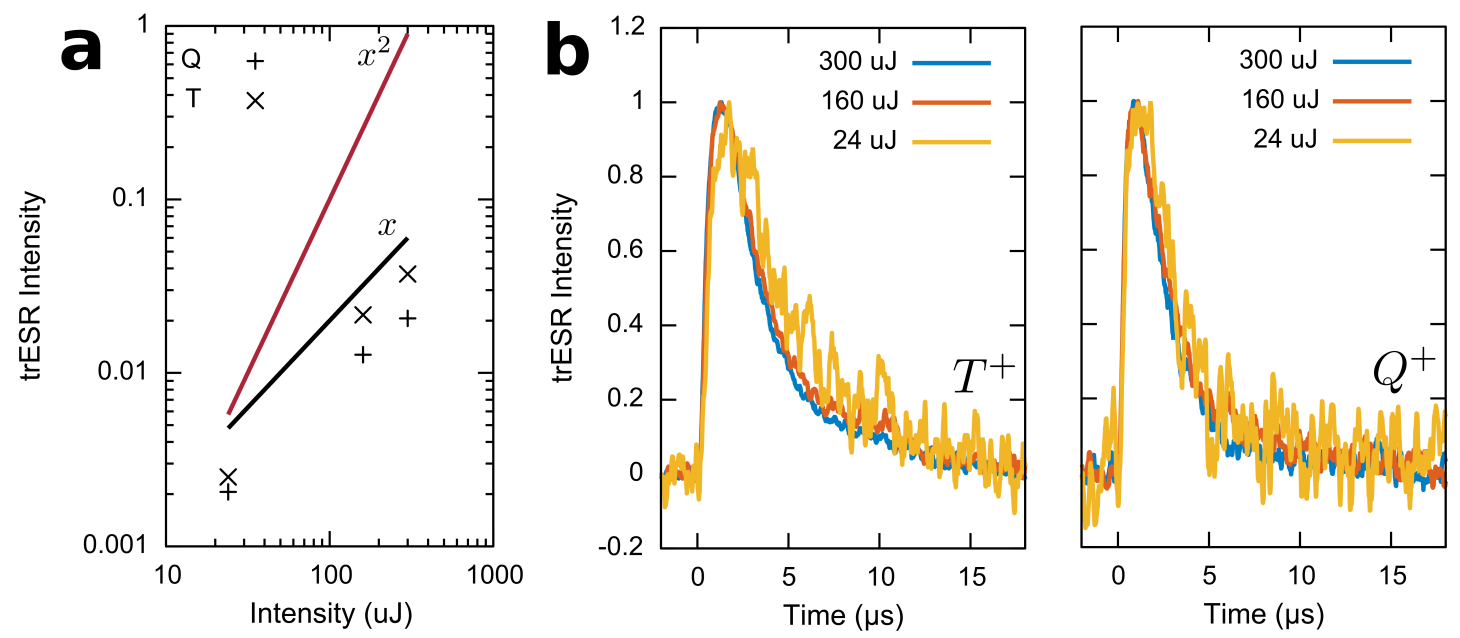

Figure 4. Fluence-dependence of trESR kinetics of TIPS-Tetracene films. (a) Peak absolute signal intensity as a function of fluence following $x^{a}$ behavior with $a \leq 1$ for both the trESR kinetics of the quintet (Q) and triplet (T) transitions shown in (b). The red line displays quadratic intensity dependence while black line displays linear behavior for reference. (b) At 10K normalized trESR kinetics display no significant early-time dependence on intensity and a slight increase in decay rate at higher laser power for both the quintet transition $\left(Q^{+}\right)$and triplet transition $\left(T^{+}\right)$.

1 Bencini, A. \& Gatteschi, D. Electron paramagnetic resonance of exchange coupled systems (Springer Science \& Business Media, 2012).

${ }^{2}$ Itoh, K. Electronic structures of aromatic hydrocarbons with high spin multiplicities in the electronic ground state. Pure and Applied Chemistry 50, 1251-1259 (1978).

3 Stoll, S. \& Schweiger, A. Easyspin, a comprehensive software package for spectral simulation and analysis in epr. J. Magn. Reson. 178, 42-55 (2006).

${ }^{4}$ Bayliss, S. L. et al. Geminate and nongeminate recombination of triplet excitons formed by singlet fission. Phys. rev. lett. 112, 238701 (2014). 